\title{
Empiema necessitatis y tuberculosis diseminada en un paciente adulto inmunocompetente: reporte de caso
}

Alejandra Bendezú Chacaltana ${ }^{1, a}$

\section{RESUMEN}

La tuberculosis diseminada es rara en el huésped inmunocompetente. Resulta de la diseminación linfohematógena de Mycobacterium tuberculosis durante la tuberculosis primaria y postprimaria.

Este es un reporte de caso de un paciente varón que ingresa al hospital por presentar signos clásicos de proceso infeccioso a nivel pulmonar y articular, además de presentar una masa en la pared torácica anterior derecha y colección pleural derecha. Pensándose que se trataba de un empiema necessitatis, una extensión de líquido pleural purulento a través de tejidos adyacentes que forman un absceso en la pared torácica, se decide su hospitalización. Empiema necessitatis es un hallazgo raro en la actualidad. Tuberculosis es la causa más común de empiema necessitatis.

Palabras claves: Empiema; tuberculosis; Mycobacterium tuberculosis; adulto (Fuente: DeCS BIREME).

\section{Empyema necessitans and disseminated tuberculosis in an immunocompetent adult patient: a case report}

\section{ABSTRACT}

Disseminated tuberculosis is a rare condition in immunocompetent hosts. It results from lymphohematogenous spread of Mycobacterium tuberculosis during both primary and postprimary tuberculosis.

This is a case report of a male patient admitted to our hospital for typical clinical signs of infectious disease affecting lungs and joints, and a right anterior chest wall mass and right-sided pleural effusion. It was thought that he had empyema necessitans, an extension of purulent pleural fluid through adjacent tissues forming an abscess on the thoracic wall, so he was hospitalized. Nowadays, empyema necessitans is a rare clinical finding. Tuberculosis is the most common cause of empyema necessitans.

Keywords: Empyema; tuberculosis; Mycobacterium tuberculosis; adult (Source: MeSH NLM).

1. Médico Internista, Asistente del Servicio de Medicina Interna $\mathrm{N}^{\circ} 2$.

a. Hospital Nacional Guillermo Almenara Irigoyen. Lima, Perú. 


\section{INTRODUCCIÓN}

Aproximadamente 9 millones de personas desarrollaron Tuberculosis (TB) activa en el 2013, de los cuales 1. 5 millones fallecieron ${ }^{(1)}$. Para el año 2015, según la Organización Mundial de la Salud, la cifra de personas que tenían TB en el mundo se estimó en 10.4 millones. TB continúa siendo una enfermedad relacionada a la pobreza, hacinamiento y malnutrición. Otros factores de riesgo son estados que condicionan inmunodepresión como infección por Virus de Inmunodeficiencia Humana $(\mathrm{VIH})$, Diabetes Mellitus, uso de alcohol y otras drogas, uso de corticoides o inmunomoduladores entre los más frecuentes ${ }^{(2)}$.

TB pulmonar es el sitio inicial de infección y puede propagarse a otros órganos. Las formas extrapulmonares suponen el $10-20 \%$ de los casos de TB en enfermos inmunocompetentes, pero este tipo de presentación se incrementa en las personas con inmunodeficiencia. Las localizaciones extrapulmonares más frecuentes son los ganglios linfáticos, el sistema osteoarticular, el aparato genitourinario, las meninges y el encéfalo. Otras presentaciones como las cutáneas, representan menos del $0.5 \%$ de los casos en los países industrializados ${ }^{(3,4)}$.

El compromiso multifocal esquelético por TB es raro, aún en áreas endémicas y puede ocurrir como resultado de diseminación hematógena de otro foco primario como ganglios linfáticos cervicales, pulmones, amígdalas o tracto gastrintestinal ${ }^{(5)}$. Otros estudios señalan que TB es causa rara de abscesos cutáneos; una de las etiologías es el empiema necessitatis, que ocurre cuando un empiema se extiende desde la pleura hasta tejidos circundantes, hoy no es tan común por el tratamiento antibiótico oportuno y el drenaje del empiema. Es más frecuente en pacientes inmunocomprometidos ${ }^{(6,7)}$.

Empiema necessitatis es una entidad clínica en la cual un empiema intratorácico se extiende a través de la pleura parietal y una debilidad de la pared torácica, formando una colección de pus en los tejidos blandos extratorácicos. Aunque es raro, puede ocurrir en el contexto de neumonías necrotizantes o abscesos pulmonares. Cuando es causado por TB, este tipo de complicación puede ocurrir en presencia de empiema tuberculoso pulmonar ${ }^{(8)}$.

Tuberculosis diseminada es rara en el huésped inmunocompetente. Los factores de riesgo que se atribuyen al desarrollo de TB extrapulmonar son fundamentalmente la edad, el sexo femenino, la existencia de infección por $\mathrm{VIH}$ y las comorbilidades del paciente como la presencia de insuficiencia renal crónica, diabetes mellitus o la existencia de inmunodepresión. La media de edad de los pacientes con TB extrapulmonar es mayor que en los pacientes con TB pulmonar ${ }^{(9)}$.

La TB diseminada tiene presentación clínica variable y los signos tempranos de infección vistos en una radiografía de tórax son inespecíficos. Sin embargo, en pacientes con factores de riesgo, la sospecha clínica es la clave para el diagnóstico de la enfermedad, así como el inicio temprano del tratamiento puede mejorar el pronóstico ${ }^{(2)}$.

En pacientes que no manifiestan la enfermedad, puede haber efectos indeseables sobre la salud del paciente y el establecimiento de salud donde se atiende. Este problema puede ser visto en áreas endémicas, donde el control de TB es difícil. El reporte de la enfermedad en el centro de salud local es necesario para tratamiento adecuado, controlado y mantenimiento de la salud pública ${ }^{(7,10)}$.

Se presenta un caso de EN como complicación rara de TB diseminada en un paciente sin antecedentes importantes y muestra el valor de la baciloscopía y el Xpert MTB/RIF como métodos diagnósticos, lo que permitió instaurar un tratamiento oportuno y adecuado. Para describir este reporte de caso, además de la evaluación clínica, se realizó una revisión de la literatura en la base de datos PubMed.

\section{CASO CLÍNICO}

Varón de 23 años, natural de la provincia de Cañete, procedente del distrito de San Juan de Lurigancho en Lima. De ocupación vendedor de celulares, soltero y católico. Ingresa por emergencia del hospital con tiempo de enfermedad de 2 semanas caracterizado por fiebre de $39^{\circ} \mathrm{C}$ todos los días, tos productiva y dificultad para respirar que se acentúa al inspirar. Una semana antes de su ingreso nota aumento de volumen de rodilla izquierda y de región mamaria derecha, ambas con dolor a la palpación que limitan movilización. Por aumentar dificultad para respirar acude al hospital donde es internado.

Antecedentes: Catorce meses antes de su ingreso sufrió quemaduras de segundo y tercer grado en las cuatro extremidades por accidente de trabajo con flama de fuego, por lo que se le realizaron autoinjertos de piel procedentes de la región torácica. Refiere haber acudido a un prostíbulo una vez, 4 años antes. No tiene historia de consumo de tabaco, alcohol ni otras drogas. Niega contacto epidemiológico con Tuberculosis, con otras enfermedades y cirugías, niega ingesta de medicamentos. No tiene otros antecedentes patológicos contributorios.

Al examen físico: Temperatura: $38.9{ }^{\circ} \mathrm{C}$, Frecuencia cardiaca: 100 latidos por minuto. Otras funciones vitales normales. Cicatrices queloides con hiperpigmentación difusa y atrofia perilesional con retracción que compromete extremidades superiores e inferiores. Cicatriz atrófica hipopigmentada en región costal derecha que se extiende hasta hipocondrio izquierdo de aproximadamente $10 \mathrm{~cm}$. de diámetro, de bordes irregulares. Aumento de volumen y flogosis en rodilla izquierda, dolorosa a la palpación. Masa localizada en pared torácica anterior derecha de 10 $\mathrm{cm}$ de diámetro a nivel submamario, fluctuante, dolorosa a la palpación (Figura 1). Murmullo vesicular abolido y crepitantes en base de hemitórax derecho. Despierto, lúcido, marcha conservada. 


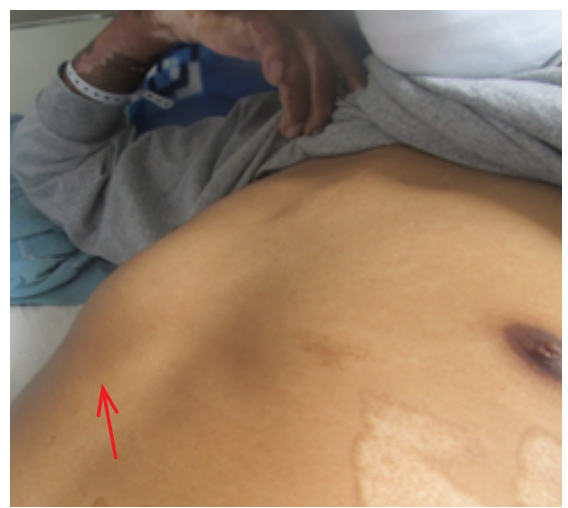

Figura 1. Masa en región submamaria derecha

Exámenes auxiliares: Los recuentos sanguíneos estuvieron dentro de límites normales. Pruebas de ELISA-HIV, HTLV1, VDRL fueron negativas. Muestra de esputo, líquido sinovial de rodilla derecha y secreción del absceso cutáneo en el tórax revelaron presencia de bacilos ácido alcohol resistente +++ y el Xpert MTB/RIF confirmó Mycobacterium tuberculosis sensible a Rifampicina.
La radiografía de tórax mostró radiopacidad basal derecha con senos obturados, impresiona tabicado (Figura 2). La tomografía de tórax reveló una masa localizada en la pared torácica anterior derecha comunicándose con el espacio pleural derecho, donde se evidencia efusión pleural (Figura 3).

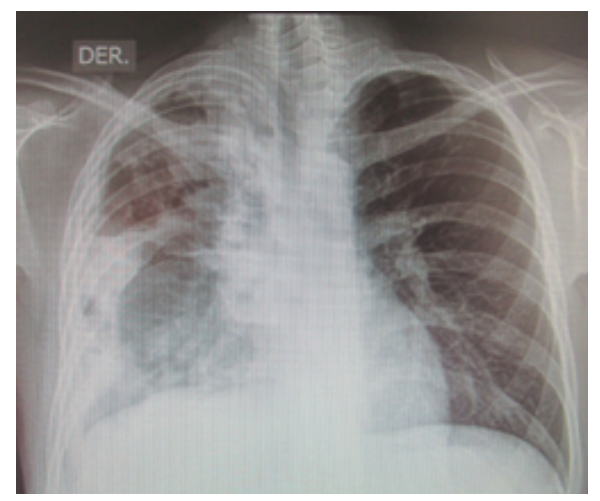

Figura 2. Radiografía de tórax al ingreso

Por el compromiso pulmonar, pleural y articular, el diagnóstico de tuberculosis diseminada fue hecho. Además de presentar empiema necessitatis como complicación de la TB pulmonar.

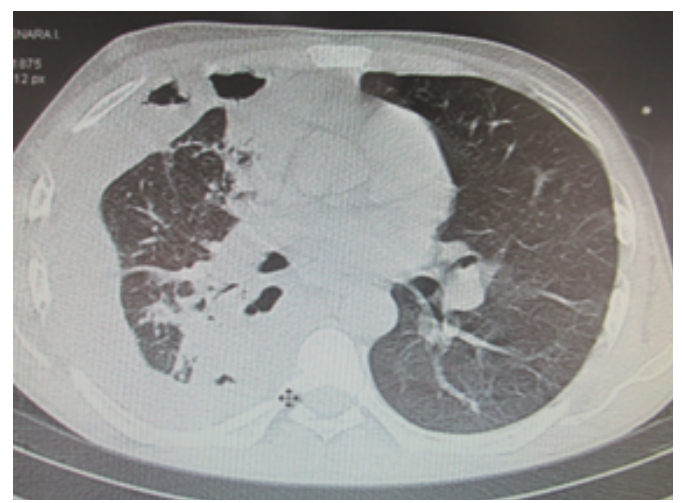

Figura 3. TAC tórax al inicio del tratamiento anti TBC 
Evolución: Se inició tratamiento con esquema I Fase I anti TB: Isoniazida 300 mg por día, Rifampicina 600 mg por día, Pirazinamida $1500 \mathrm{mg}$ por día y Etambutol $1200 \mathrm{mg}$ por día. La evaluación por los especialistas en Cirugía de tórax consideró que no requería drenaje de empiema.

Presenta evolución clínica y radiológica favorable de afección pulmonar y articular, siendo dado de alta al cumplir 5 meses de tratamiento, cuando se evidenció en una tomografía torácica de control que el empiema estaba casi resuelto. Completó 6 meses de tratamiento con controles ambulatorios y resolución completa del cuadro.

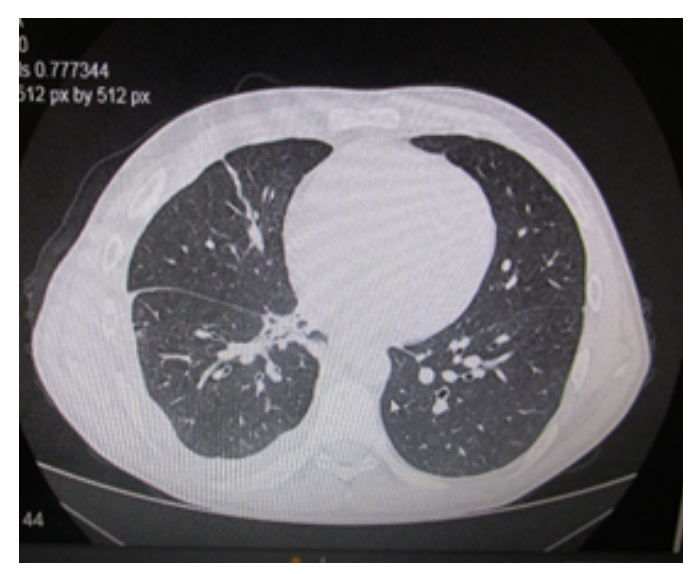

Figura 4. TAC tórax 5to mes de tratamiento antiTBC

\section{DISCUSIÓN}

La TB constituye una amenaza para la salud pública mundial, aún con los esfuerzos conjuntos que se hacen para controlarla. Cuando se habla de TB diseminada las tasas de morbilidad y mortalidad asociadas a esta, se incrementan. TB diseminada suele asociarse estados de inmunodeficiencia. Hay pocos casos reportados de TB diseminada en inmunocompetentes en el mundo. A pesar de ser una Patología no muy común es de relevancia por la cantidad de pacientes con TB, asimismo por que podría ser subdiagnosticada, generando graves repercusiones negativas sobre la salud pública, especialmente en países pobres.

En Perú, según el análisis de la situación epidemiológica de la tuberculosis 2015 del Ministerio de Salud, la enfermedad se concentra en los departamentos de la costa central y la selva. En Lima, la incidencia más alta de TB se concentra en los distritos de San Juan de Lurigancho, Ventanilla, El Agustino, Ate, Cercado, La Victoria, San Juan de Miraflores, Chorrillos, Santa Anita, Rímac e Independencia, con más de 75 casos por 100000 habitantes.

Nuestro paciente no tenía factores de riesgo ni antecedentes contributorios. Resulta importante notar que procedía de uno de los distritos con tasa elevada de $\mathrm{TB}$, información que no es presentada en reportes de casos hallados en la literatura ${ }^{(6,7,8)}$.

Clínicamente, algunos, reportes ${ }^{(6,7,8)}$ describen empiema necessitatis como complicación tardía de TB pulmonar. Aquí es necesario agregar que EN también ha sido descrito como masas torácicas múltiples. Sin embargo hay un reporte ${ }^{(10)}$ de rápida aparición de los síntomas y la presencia concomitante de una masa en la pared torácica compatible con empiema necessitatis.

Hay reportes donde $E N^{(8)}$ fue la única manifestación clínica. Nuestro paciente presentó otra sintomatología, además de empiema necessitatis, como también el caso reportado por Babamahmoodi y colaboradores ${ }^{(10)}$.

Otros reportes señalan como inmunocompetentes los casos presentados, aunque sí tenían antecedentes que condicionan riesgo para la salud como Diabetes Mellitus, Hipertensión arterial, Alzheimer, antecedente de TB, tabaco, uso de drogas ilícitas en el pasado y haber estado en la cárcel ${ }^{(8,10)}$.

En todos los casos revisados se evidenció Mycobacterium tuberculosis en los cultivos de tejidos comprometidos y buena respuesta al tratamiento instaurado.

Hubo 2 casos ${ }^{(8,10)}$ que de forma igual a nuestro reporte, solo recibieron tratamiento médico. No se sometieron a ninguna opción quirúrgica y su evolución fue desfavorable. Los estudios reportan que el tratamiento médico deber ser concomitante al tratamiento quirúrgico (drenaje de empiema), pero el caso reportado ahora, describe la evolución favorable sin tratamiento quirúrgico.

En algunos países desarrollados el diagnóstico de TB puede ser difícil, se requiere alta sospecha clínica y exámenes radiológicos, microbiológicos e histopatológicos para 
lograr un diagnóstico. En países en vías de desarrollo, e diagnóstico puede sospecharse rápidamente pero éste caso resalta la importancia de considerar TB diseminada en pacientes que viven en áreas endémicas como Perú.

\section{REFERENCIAS BIBLIOGRÁFICAS}

1. Brigden G, Hewison C, Varaine F. New developments in the treatment of drug-resistant tuberculosis: clinical utility of bedaquiline and delamanid. Infect Drug Resist. 2015; 8: 367-378.

2. Hilal T, Hurley P, McCormick M. Disseminated tuberculosis with tuberculous meningitis in an immunocompetent host. Oxf Med Case Reports. 2014; 2014(7): 125-8.

3. Marco A, Solé R, Raguer E, Aranda M. Goma o absceso tuberculoso metastásico como diagnóstico inicial de tuberculosis en un paciente inmunocompetente: una presentación inusual. Rev esp sanid penit.2014; 16(2): 59-62.

4. Hatice S, Hatice I, Sevill, Hakan T, Muammer B. Disseminated tuberculosis in a non immune compromised patient with a complicated diagnosis. Respir Med Case Rep. 2015; 14: 1-3.

5. Ramos P, Ramos H, Lopes F, Medeiros Z, Maciel M, Florencio R et al. Multifocal skeletal tuberculosis in an immunocompetent patient: a case report. BMC Infect Dis. 2015; 15:235.

6. Scott K, Fidaa S, Derek F, Jinesh M. Empyema Necessitatis. Chest. 2010; 138: 1-2.

7. Magness D. Empyema necessitans caused by Mycobacterium tuberculosis in an immunocompetent patient. WMJ. 2013;112(3):129-30.
8. Mendes M, Alves M, Bernardes J, Santos L. Empyema necessitans: very late complication of pulmonary tuberculosis. BMJ Case Rep. 2013.

9. Ramirez M, MenéndezA, Noguerado A. Tuberculosis extrapulmonar, una revisión. Rev Esp Sanid Penit. 2015; 17(1):3-11.

10. Babamahmoodi F, Davoodi L, Sheikholeslami R, Ahangarkani F. Tuberculous Empyema Necessitatis in a 40-Year-Old Immunocompetent Male. Case reports in infectious diseases. 2016:2016; 4

Fuentes de financiamiento:

El artículo ha sido financiado por la autora.

Conflictos de interés:

La autora declara no tener ningún conflicto de interés.

Correspondencia:

Nombre: Alejandra Bendezú Chacaltana

Dirección: Pasaje Ideal N187. Departamento 401. Jesús María. Lima. Teléfono: 965303513

Correo electrónico: abendezuch@hotmail.com

Recibido: 05 de abril de 2017
Evaluado: 12 de mayo de 2017
Aprobado: 23 de agosto de 2017

(c) La revista. Publicado por Universidad de San Martín de Porres, Perú. (cc) $\mathbf{B Y}$ Licencia de Creative Commons Artículo en acceso abierto bajo términos de Licencia Creative Commons Atribución 4.0 Internacional. (http://creativecommons.org/licenses/by/4.0/) 